https://helda.helsinki.fi

\title{
Taste compound - Nanocellulose interaction assessment by fluorescence indicator displacement assay
}

\section{Manninen, Hanna}

2020-07-15

Manninen , H , Durandin , N , Hopia , A , Vuorimaa-Laukkanen , E \& Laaksonen , T 2020 , '

Taste compound - Nanocellulose interaction assessment by fluorescence indicator displacement assay ' , Food Chemistry , vol. 318 , 126511 . https://doi.org/10.1016/j.foodchem.2020.126511

http://hdl.handle.net/10138/327223

https://doi.org/10.1016/j.foodchem.2020.126511

cc_by_nc_nd

acceptedVersion

Downloaded from Helda, University of Helsinki institutional repository.

This is an electronic reprint of the original article.

This reprint may differ from the original in pagination and typographic detail.

Please cite the original version. 
Taste compound - nanocellulose interaction assessment by fluorescence indicator displacement assay

Hanna Manninen ${ }^{a *}$, Nikita Durandin ${ }^{\mathrm{a}}$, Anu Hopia ${ }^{\mathrm{b}}$, Elina VuorimaaLaukkanen $^{\mathrm{a}}$, Timo Laaksonen ${ }^{\mathrm{a}, \mathrm{c}}$

Affiliations

6

*Correspondance: hanna.t.manninen@tuni.fi

a: Tampere University, Faculty of Engineering and Natural Sciences, FI8 33014 Tampere University, Finland b: University of Turku, Functional Foods Forum, FI-20014 Turku, Finland c: Drug Research Program, Division of Pharmaceutical Biosciences, Faculty of Pharmacy, University of Helsinki, FI-00014 University of Helsinki, 12 Finland 


\section{Abstract}

Interactions between taste compounds and nanofibrillar cellulose were studied. For this, a new fluorescent indicator displacement method was developed. Two fluorescent indicators, namely, Calcofluor white and Congo red, were chosen because of their specific binding to cellulose and intrinsic fluorescence. Seven taste molecules with different structures and properties were successfully measured together with NFC and ranked according to their binding constants. The most pronounced interactions were found between quinine and nanofibrillar cellulose $\left(1.4 \times 10^{4} \mathrm{M}^{-1}\right)$ whereas sucrose, aspartame and glutamic acid did not bind at all. Naringin showed moderate binding while stevioside and caffeine exhibited low binding. The comparison with microcrystalline cellulose indicates that larger surface area of nanofibrillated cellulose enables stronger binding between the binder and macromolecules. The developed method can be further utilized to study interactions with different compound classes with nanocellulose materials for purposes of food, pharmaceutical and dye industries using a conventional plate reader in a highthroughput manner. 


\section{Keywords}

Nanofibrillar cellulose; fluorescence indicator displacement assay; taste

34 
Nanocellulose materials represent a class of cellulose materials with at least one nanoscale dimension produced either with enzymatic, chemical or physical methods from natural cellulose fibers (Klemm, Kramer, Moritz, Lindström, Ankerfors, \& Gray, 2011). Nowadays they have many uses for example as bioabsorbent in wastewater treatment and in biomedical applications, drug delivery systems, tissue engineering and wound dressings as a food additive was one of the first applications proposed (Turbak, Snyder, $\&$ Sandberg, 1983a). High surface area and aspect ratio, suitable rheological behaviour (high viscosity even at low concentrations) and the easiness of chemical modifications are advantageous for the applications in food industry, particularly in food packaging (Gómez et al., 2016; Klemm et al., 2011).

Since 1980s, many food related applications utilizing nanocellulose have been developed. In a review by Gómez et al. (2016) the applications in food science were divided in three groups: 1) as a food stabilizer, 2) as a functional food ingredient, and 3) in food packaging. As a stabilizing agent, nanocellulose materials have been used in various different food products such as in fat and oil containing products (gravies, salad dressings, and whipped toppings) (Turbak, Snyder, \& Sandberg, 1982, 1983a, 1983b). Furthermore, it has been used in this purpose to prevent the spreading of cookie fillings (Kleinschmidt, 1988), to improve the shape retention of frozen desserts (Yano, Abe, Kase, Kikkawa, \& Onishi, 2012) and most recently, in the shape retention of ice cream (Velásquez-Cock, et al., 2019). In functional 
foods, nanocellulose materials have been used in low-calorie applications in products with high-energy content such as hamburgers (Ström, Öhgren, \& Ankerfors, 2013) and to replace fats in food formulations and thus reduce their energy density (Cantiani, Knipper, \& Vaslin, 2002). Furthermore, nanocellulose materials have showed promising characteristics as dietary fibers (Andrade, Mendonça, Helm, Magalhães, Muniz, \& Kestur, 2015). friendly option to fossil fuel based and non-biodegradable materials (Azeredo, Rosa, \& Mattoso, 2017). Nanocellulose materials can act as high air and oxygen barrier, which makes them competitive to other packaging materials (Aulin, Gällstedt, \& Lindström, 2010; Gómez et al., 2016). They can also serve as carriers for active substances in food packaging applications (Huq et al., 2012). For example, Lavoine, Desloges and Bras (2014) used a paper coated with microfibrillated cellulose for the controlled release of caffeine, whereas Jipa, Stoica-Guzun and Stroescu (2012) studied controlled release of sorbic acid from bacterial cellulose films.

In this study, the aim was to evaluate the interactions between nanocellulose materials and taste compounds. Despite the many food related applications of nanocellulose materials, to our knowledge there are no systematic studies about the possible effects of nanocellulose to the taste of food. Troszyńska et al. (2010) studied the effect of food gums (i.e. guar, xanthan, arabic) and carboxymethylcellulose (CMC) on the astringency induced by phenolic compounds. According to their study, CMC was the best at masking astringency. Furthermore, the interactions between nanocellulose materials and drug molecules have been studied. Particularly, Kolakovic et al. (2013) 
used isothermal titration calorimetry (ITC) and an incubation method (incubation of drug molecules with NFC, centrifugation and quantification of an unbound drug from supernatant) to study the binding of drug compounds to nanofibrillated cellulose (NFC). In similar manner, Jackson, Letchford, Wasserman, Ye, Hamad, \& Burt (2011) studied the binding of drug molecules to nanocrystalline cellulose (NCC) by measuring the amount of unbound molecules by using a spectrophotometry method.

The methodologies presented above are accurate, but time-consuming and molecule dependent. For each compound, a new or at least refined methodology is needed. In contrast, a more generic method based on fluorescent indicator (FI) displacement for nanocellulose-taste compound interaction assessment is developed in this study. With this method, it is possible to screen a wide spectrum of molecules with different characteristics with one method using a plate reader with e.g. a 96-well plates. Thus, the developed method is both affordable and efficient. The method is based on the competitive binding of a well-known FI molecule and a second molecule, whose binding to a macromolecule, in this case to NFC, is investigated. If the interaction between molecule of interest and NFC occurs, a decrease of FI fluorescence intensity can be detected as it is displaced from the fiber surface. Similar methods have been used before for example in the assessment interactions of different analytes to DNA, RNA and proteins (Asare-Okai, \& Chow, 2011; Ham, Winston, \& Boger, 2003; Mock. Langford, Dubois, Criscimagna, \& Horowitz, 1985; Zhang, Umemoto, \& Nakatani, 2010). These methodologies have been reviewed by Nguyen \& Anslyn (2006) and Tse, \& Boger, 2004. Nevertheless, to our knowledge these methods have not 
been used before to assess macromolecule interactions with taste compounds. Two FIs were chosen based on their specific binding to cellulose (Wood, 1980) and different photophysical properties to avoid a possible situation where the molecule of interest absorbs light at the same wavelength that is used to excite the FI. Calcofluor white has its absorption maximum at around $350 \mathrm{~nm}$ while the absorption maximum of Congo red is at around $500 \mathrm{~nm}$ (Wood, 1980). With these indicators, a wide variety of taste compounds with different taste characteristics could be studied. Seven taste compounds, caffeine, aspartame, quinine, stevioside, sucrose, naringin and glutamic acid,

\section{Materials and Methods}

\subsection{Materials}

Cellulose nanofibrils (dimeric unit presented in the Figure 1a) were obtained from UPM Corporation (Finland) as a $1.5 \mathrm{wt} \%$ hydrogel. Microfibrillated cellulose (MCC, Avicel®, Sigma-Aldrich) was used as a $1.5 \mathrm{wt} \%$ suspension prepared with water purified with a Milli-Q system (Millipore, Burlington, Massachusetts, USA). The FIs used were Fluorescence brightener 28 (Calcofluor white M2R) (Figure 1b) from Sigma-Aldrich (St. Louis, MO, USA) and Congo Red (> $98 \%$ ) from Tokyo Chemical Industry CO., LTD. (Tokyo, Japan) (Figure 1c). 
The studied taste compounds (Figure $1 \mathrm{~d}-\mathrm{j}$ ) were caffeine (99\%), naringin and aspartame (98\%) from ThermoFisher GmbH (Kandel, Germany) and glutamic acid (99\%), stevioside, sucrose (> $99 \%)$ and quinine (99\%) from Acros Organics (Geel, Belgium). The compounds were chosen based on their known taste properties to include compounds, which either create a pleasant taste (sweet and umami) or have related unpleasant characteristics (bitter).

\subsection{Methods}

\subsubsection{UV-Vis characterizations}

Water solutions of the fluorescent indicators i.e. calcofluor white (CFW) and Congo red (CR) were measured with UV-Vis-NIR spectrophotometer (UV3600, Shimadzu) in $1 \mathrm{~cm}^{2}$ standard quartz cuvettes. Absorption spectra were measured from 250 to $600 \mathrm{~nm}$ varying the concentration from 0 to $26 \mu \mathrm{M}$ for both calcofluor white and Congo Red. MQ-water was used to adjust the samples concentrations. Absorption maxima were detected at $349 \mathrm{~nm}$ and 499 $\mathrm{nm}$ for $\mathrm{CFW}$ and $\mathrm{CR}$, respectively. Molar extinction coefficients were calculated based on the absorption measurements.

\subsubsection{Titration of the fluorescent indicator with nanofibrillar cellulose}

Fluorescent indicators CFW and CR in concentrations of $6 \mu \mathrm{M}$ and $2.5 \mu \mathrm{M}$, respectively, were titrated with a NFC hydrogel to a final NFC concentration of $0.04 \mathrm{M}$. The concentration of FIs were chosen to avoid inner filter effects on the fluorescence of FIs. As the molecular weight of NFC macromolecules varies, the concentration of NFC is represented in moles of monomeric cellulose units per liter using $162.14 \mathrm{~g} / \mathrm{mol}$ as the molar mass of the monomer. This practice is used commonly with biopolymers such as DNA and RNA 
where the concentration is expressed as the concentration of nucleobases or pairs of nucleobases. The changes in the fluorescence intensity of CFW upon titration with NFC were measured in triplicates by spectrofluorometer Fluorolog-3® (Jobin Yvon) or plate reader Fluoroskan Ascent FL (Thermo Labsystems). The changes in the fluorescence intensity of CR upon titration with NFC were measured in triplicates by using a plate reader. The excitation/emission filter pairs for measurement with spectrofluorometric plate reader were chosen to be $355 / 460 \mathrm{~nm}$ for CFW and $485 / 590 \mathrm{~nm}$ with $\mathrm{CR}$ based on their absorption/emission spectra. The titration of MCC with $\mathrm{CR}$ was conducted in a similar manner as with NFC with concentration range from 0.002 to $0.088 \mathrm{M}$. MCC concentration was estimated in the same way as for NFC.

The binding constants $\left(K_{\text {bind }}\right)$ for FIs with NFC were calculated using BenesiHildebrand method (Benesi \& Hildebrand, 1949) as follows:

$\frac{I_{\text {max }}-I_{\text {free }}}{I_{n}-I_{\text {free }}}=1+\frac{1}{K_{\text {bind }}[N F C]}$

where $[\mathrm{NFC}]$ is the added NFC concentrations, $I_{\max }$ is the maximum fluorescence intensity of FI in the presence of NFC when the saturation is reached, $I_{\text {free }}$ is the fluorescence intensity of FI in the absence of NFC, $I_{n}$ is the fluorescence intensity of FI in the presence of NFC at an intermediate concentration and $K_{\text {bind }}$ is the binding constant for the FI. By plotting $\frac{I_{\text {max }}-I_{\text {free }}}{I_{n}-I_{\text {free }}}$ versus $1 /[\mathrm{NFC}]$ the values of $K_{\text {bind }}$ were obtained from the slope of the linear fit. 

complex with taste compounds

All the samples contained either $0.04 \mathrm{M}$ of NFC with $6 \mu \mathrm{M}$ CFW or $0.025 \mathrm{M}$ of NFC with $2.5 \mu \mathrm{M}$ CR and varying concentrations of the taste compounds (Table 1). The concentration ranges for the taste compounds were chosen based on their solubility in water. All solutions were mixed carefully to avoid bubbles. FI for each compound was chosen based on their photophysical characteristics, i.e. whether they would absorb light at the excitation wavelength of the FI or not. In order to estimate possible errors by using different FIs, cross-validation of caffeine-NFC interaction was studied by using both CFW and CR. $150 \mu \mathrm{L}$ of each sample solution was pipetted on a well plate and measured with plate reader as above (2.2.2.). Each taste compound was studied as triplicates. before. As the substitution of FI causes decreasing fluorescence intensity, equation 1 was modified as follows:

$\frac{I_{0}-I_{\text {free }}}{I_{0}-I_{n}}=1+\frac{1}{K_{\text {bind }}[T C]}$ [TC] is the added taste compound concentrations, $K_{\text {bind }}$ is the binding constant of the taste compound, $I_{0}$ is the fluorescence intensity of FI-NFC mixture in the absence of the taste compounds, $I_{\text {free }}$ is the fluorescence intensity of FI in the absence of NFC, $I_{n}$ is the fluorescence intensity of FINFC mixture in the presence of the taste compounds at an intermediate concentration. 


\subsubsection{Cross-validation with ITC}

Isothermal titration calorimetry was performed using a Microcal VP-ITC (GE Healthcare, Life Sciences, MicroCal, Northampton,MA). A sample cell was filled with quinine $(0.39 \mathrm{mM})$. Experiments were carried out at $25{ }^{\circ} \mathrm{C}$ by injecting $20 \mu \mathrm{L}$ of $15 \mathrm{mM}$ NFC sample solution 15 times. As control measurements, MQ was titrated with $15 \mathrm{mM}$ NFC and $0.39 \mathrm{mM}$ quinine with MQ. The differential enthalpy curves of heat of titration of MQ with NFC and the averaged enthalpy of titration of quinine with MQ were then subtracted from the curves of binding of quinine to NFC. Data analysis was performed with Microcal Origin software and one binding site model was used for fitting.

\section{Results and Discussion}

\subsection{Binding constants of fluorescence indicators}

Based on the results of spectrophotometry, the molar extinction coefficients in water for CFW and CR were calculated to be $\varepsilon_{\mathrm{CFW}}(349 \mathrm{~nm})=53.2 \times 10^{3} \mathrm{M}^{-}$ ${ }^{1} \mathrm{~cm}^{-1}$ and $\varepsilon_{\mathrm{CR}}(499 \mathrm{~nm})=38.9 \times 10^{3} \mathrm{M}^{-1} \mathrm{~cm}^{-1}$. Thus, indicator concentrations of $6 \mu \mathrm{M}$ and $2.5 \mu \mathrm{M}$ for $\mathrm{CFW}$ and $\mathrm{CR}$ were used for the titration experiments with NFC to neglect the inner filter effect on the fluorescence. During the titrations of CFW and CR with NFC an increase in the fluorescence intensity of both dyes was observed, indicating that both FIs bind to NFC. The fluorescence intensities of CFW and CR against the added NFC concentration are presented in Figure 2. The data is also presented according to Eq. (1) to calculate the binding constants (Figure 2, insets). 
The obtained binding constants were $27 \pm 7 \mathrm{M}^{-1}$ for $\mathrm{CFW}$ and $58 \pm 12 \mathrm{M}^{-1}$ for CR. Based on the saturation curves (Figure 2), NFC concentrations of $0.04 \mathrm{M}$ with CFW and $0.025 \mathrm{M}$ with $\mathrm{CR}$ were chosen for taste compound titrations as the saturation and maximum intensity were reached at these concentrations. When MCC was titrated with $\mathrm{CR}$, saturation was not reached within the studied concentration range and the binding constant was estimated to be approximately $4 \mathrm{M}^{-1}$. This is more than 10 times lower compared to NFC-CR interaction and is probably due to the considerably lower specific

\subsection{Binding constants of taste compounds}

The interaction between the pre-formed NFC-FI complexes and taste compounds resulted in a clear decrease in the fluorescence of the FIs because of FI displacement from the NFC matrix. As the FI-NFC complexes have stronger fluorescence that the free FIs, the overall fluorescence intensity in the system will decrease if the dyes are released from the NFC surface. This happens when a taste compound binds to a cellulose surface that is initially fully covered by the FI. It is good to notice that the measured signal comes from the FI in all the measurements, and not from the taste compounds. FI displacement curves are presented in Figure 3 for those taste compounds that 
showed clear complex formation with NFC. These curves can be used to

evaluate the binding constants according to eq. (2).

Clear trends in the fluorescence intensity can be seen in Figure 3 for caffeine, stevioside, naringin and quinine even though the experimental fluctuations are considerable especially for stevioside and naringin. The binding isotherms can be used for ranking the taste compounds in the order of binding strength and for the evaluation of the binding constants. As an example, a $20 \%$ FI (CFW) displacement was achieved at ca. $5 \mathrm{mM}$ concentration of caffeine, whilst for quinine the same percent was achieved at ca. $0.025 \mathrm{mM}$ concentration. Roughly $1.8 \mathrm{mM}$ and $0.2 \mathrm{mM}$ concentrations for stevioside and and naringin respectively were needed to reach the same displacement. Sucrose, aspartame and glutamic acid had negligible binding according to our measurements, as no clear fluorescence decrease was seen with these molecules. Finally, the binding constants of all the tested compounds calculated with Eq. (2) are presented in Table 1 as mean values of triplicates.

In order to verify the comparability of the results obtained by different FIs, the binding values for caffeine was estimated with both indicators. The slopes for caffeine-NFC interactions plotted according to Eq. (2) are close to each other. The calculated binding constant of caffeine to NFC obtained with CR was $86 \mathrm{M}^{-1}$, which is very close to the measured binding constant with $\mathrm{CFW}$ (70 $\mathrm{M}^{-1}$, Table 1). Thus, the method can be used with either of the selected fluorescence indicators, and the indicator can be chosen based on whether the molecules have spectral overlap with the FI or not. As all seven taste compounds with different structures and properties were measured 
manner, it can be concluded that a similar methodology can be also utilized in future for studying larger sets of compounds for applications in e.g. food industry and pharmaceutical fields.

Isothermal titration calorimetry (ITC) was used for cross-validation of the method. As only relatively high enthalpy changes can be measured with this method, quinine with the highest binding constant to NFC was chosen for these studies. Enthalpy curve of quinine binding to NFC resulting from ITC is presented on Figure 4. The estimated binding constant for quinine measured with ITC and calculated with one binding sites model was 19000 $\pm 5790 \mathrm{M}^{-1}$. Based on these results it can be concluded that the binding constants achieved with fluorescence indicator displacement method are reasonably accurate and in line with results obtained with a more established ITC methods. Furthermore, with this method weaker interactions can be measured than with traditional methods like ITC.

Based on estimated binding constants, taste compounds can be divided into four groups: non-binding molecules, molecules with weak interactions, molecules with moderate interactions and molecules with distinct interactions. Of the studied compounds, sucrose, aspartame and glutamic acid belong to the group of non-binding molecules, caffeine and stevioside have weak interactions, whereas naringin has moderate interactions. Quinine has clearly more pronounced interactions than the other studied molecules, with ca. 200 times higher binding constant than caffeine for example, making it the strongest binding molecule in our test set. The measured binding constant $\left(14000 \mathrm{M}^{-1}\right.$ ) is of the same order of magnitude as was measured for 
hydrophobin proteins binding to NFC (Kolakovic, et al., 2013), indicating strong binding between quinine and NFC.

From the Table 1 it can be seen, that the interactions seem to partly correlate with the aqueous solubility and octanol/water partition of the compounds. The highest binding constant was achieved with quinine, which is also the least water soluble of the studied molecules. This indicates that despite NFC hydrophilic nature, in aqueous solutions the nanofilbrillar cellulose is acting as a slightly hydrophobic target as water molecules already occupy most of its surface. Also, all the non-binding molecules have relatively high solubilities and $\operatorname{low} \log \mathrm{P}$ values. However, caffeine with $\operatorname{lower} \log \mathrm{P}$ and higher solubility has higher binding constant compared to aspartame. This might be explained by the negative charge of aspartame as well as glutamic acid in aqueous solutions (near neutral or slightly acidic conditions) lowering the probability of binding to nanofibrillar cellulose, which contains some amount of negatively charged hemicellulose on its surface (Kolakovic et al., 2013). Indeed, slightly negative zeta-potential values for NFC at pH 5 have been previously reported (Fall, Lindström, Sundman, Ödberf \& Wågberg, 2011). Furthermore, Kolakovic et al. (2013) stressed the stronger interaction of NFC with positively charged drugs in comparison to neutral or anionic drug molecules, as the electrostatic interactions have a significant impact on complex formation. On the other hand, quinine with the highest binding constant has a positive charge in this $\mathrm{pH}$ favoring the binding. Also, the amine groups might increase the binding probability in the case of quinine and caffeine. Furthermore, it is probable that also other effects, such as hydrogen 
bonding ability of the compounds, planarity and steric hindrances effect the binding.

Based on our results, the bitter tasting molecules are top-ranked in terms of their NFC binding constants. This finding indicates that NFC might be used as bitterness suppressing material in the future. Noteworthy, it has been already shown that CMC is able to mask the astringent taste of phenolic compounds (Troszyńska et al., 2010). NFC can be expected to have similar

\section{Conclusions}

A high-throughput screening method utilizing a plate-reader was developed for the estimation of binding constants of taste molecules with NFC. In this study, binding constants between $70 \mathrm{M}^{-1}$ and $14000 \mathrm{M}^{-1}$ were measured with good accuracy. The method seems promising for looking at the binding of 
taste compounds but also as a generic interaction assay. The studied taste compounds were divided into four groups based on their interaction strengths. Non-binding molecules were sucrose, aspartame and glutamic acid. Caffeine and stevioside were weak binders whereas naringin was a moderate NFC ligand. The bitter tasting quinine was the strongest binder in the set of the molecules studied. The magnitudes of the binding strengths seem to be at least partly correlated to the hydrophobicity of compounds. As the bitter tasting compounds are among the best NFC binders in the set, the finding can be usefull for the development of bitter suppressing or masking applications both in food and pharmaceutical industries. This should be further studied with sensory analysis to evaluate the real effects of these interactions on perceived taste.

Abbreviations

nanofibrillar cellulose

FI

fluorescence indicator

362

CR

congo red

calcofluor white

Milli-Q water

\section{Acknowledgements}

Hanna Manninen acknowledges the funding from the Finnish Cultural Foundation for funding the studies. Ph.D. Juha Määttä is acknowledged for the help in ITC studies. Timo Laaksonen acknowledges funding from the 
Academy of Finland by the profiling action on Matter and Materials, grant no. 318913 .

\section{References}

Andrade, D. R. M., Mendonça, M. H., Helm, C. V., Magalhães, W. L., de Muniz, G. I. B., \& Kestur, S. G. (2015). Assessment of nano cellulose from peach palm residue as potential food additive: part II: preliminary studies. Journal of food science and technology, 52(9), 5641-5650.

Ardizzone, S., Dioguardi, F. S., Mussini, T., Mussini, P. R., Rondinini, S., Vercelli, B., \& Vertova, A. (1999). Microcrystalline cellulose powders: structure, surface features and water sorption capability. Cellulose, 6(1), 5769.

Asare-Okai, P. N., \& Chow, C. S. (2011). A modified fluorescent intercalator displacement assay for RNA ligand discovery. Analytical biochemistry, 408(2), 269-276.

Aulin, C., Gällstedt, M., \& Lindström, T. (2010). Oxygen and oil barrier properties of microfibrillated cellulose films and coatings. Cellulose, 17(3), $559-574$.

Azeredo, H. M., Rosa, M. F., \& Mattoso, L. H. C. (2017). Nanocellulose in bio-based food packaging applications. Industrial Crops and Products, 97, 664-671.

Benesi, H. A., \& Hildebrand, J. H. J. (1949). A spectrophotometric investigation of the interaction of iodine with aromatic hydrocarbons. Journal of the American Chemical Society, 71(8), 2703-2707. Cantiani R., Knipper M., \& Vaslin S. (2002). Use of cellulose microfibrils in dry form in food formulations. United States Patent No. 6485767B1. 
Cargill, Inc. (2010) Cyclodextrin inclusion complexes and methods of

preparing same. United States Patent No. 20100160623

Dreisewerd, B., Merz, J., \& Schembecker, G. (2015). Determining the solute-

solid interactions in phytoextraction. Chemical Engineering Science, 134, 287-296.

Fall, A. B., Lindström, S. B., Sundman, O., Ödberg, L., \& Wågberg, L. 400 (2011). Colloidal stability of aqueous nanofibrillated cellulose dispersions. Langmuir, 27(18), 11332-11338.

Furia, T.E. (ed.). CRC Handbook of Food Additives. 2nd ed. Volume 2. Boca

Grignon, J., \& Scallan, A. M. (1980). Effect of pH and neutral salts upon the swelling of cellulose gels. Journal of Applied Polymer Science, 25(12), 28292843.

Gómez, C., Serpa, A., Velásquez-Cock, J., Gañán, P., Castro, C., Vélez, L., \& Zuluaga, R. (2016). Vegetable nanocellulose in food science: A review. Food Hydrocolloids, 57, 178-186.

Ham, Y. W., Winston, C. T., \& Boger, D. L. (2003). High-resolution assessment of protein DNA binding affinity and selectivity utilizing a fluorescent intercalator displacement (FID) assay. Bioorganic \& medicinal chemistry letters, 13(21), 3805-3807.

Hansch, C., Leo, A., \& Hoekman, D. (1995). Exploring QSAR.

Hydrophobic, electronic, and steric constants. ACS Professional Reference Book. ACS, Washington.

Huq, T., Salmieri, S., Khan, A., Khan, R. A., Le Tien, C., Riedl, B., Fraschini, C., Bouchard, J., Uribe-Calderon, J., Kamal, M. R., \& Lacroix, M. (2012). 
Nanocrystalline cellulose (NCC) reinforced alginate based biodegradable nanocomposite film. Carbohydrate polymers, 90(4), 1757-1763. Jackson, J. K., Letchford, K., Wasserman, B. Z., Ye, L., Hamad, W. Y., \& Burt, H. M. (2011). The use of nanocrystalline cellulose for the binding and

Jipa, I. M., Stoica-Guzun, A., \& Stroescu, M. (2012). Controlled release of sorbic acid from bacterial cellulose based mono and multilayer antimicrobial films. LWT-Food Science and Technology, 47(2), 400-406.

Kleinschmidt, D., Roberts, B.A., Fuqua, D.L. \& Melchion, J.R. (1988). Filling-containing, dough-based products containing cellulosic fibrils and microfibrils. United States Patent No. 4774095A.

Klemm, D., Kramer, F., Moritz, S., Lindström, T., Ankerfors, M., \& Gray, D. (2011) Nanocelluloses: a new family of nature-based materials. Angewandte Chemie International Edition, 50(24), 5438-5466

Kolakovic, R., Peltonen, L., Laukkanen, A., Hellman, M., Laaksonen, P., Linder, M. B., Hirvonen, J., \& Laaksonen, T. (2013). Evaluation of drug interactions with nanofibrillar cellulose. European Journal of Pharmaceutics and Biopharmaceutics, 85(3), 1238-1244.

Lavoine, N., Desloges, I., \& Bras, J. (2014). Microfibrillated cellulose coatings as new release systems for active packaging. Carbohydrate polymers, $103,528-537$.

Mazzobre, M. F., Román, M. V., Mourelle, A. F., \& Corti, H. R. (2005).

Octanol-water partition coefficient of glucose, sucrose, and trehalose.

Carbohydrate research, 340(6), 1207-1211. 
Missoum, K., Belgacem, M., \& Bras, J. (2013). Nanofibrillated cellulose surface modification: a review. Materials, 6(5), 1745-1766.

Mock, D. M., Langford, G., Dubois, D., Criscimagna, N., \& Horowitz, P. (1985). A fluorometric assay for the biotin-avidin interaction based on displacement of the fluorescent probe 2-anilinonaphthalene-6-sulfonic acid. Analytical biochemistry, 151(1), 178-181.

Nguyen, B. T., \& Anslyn, E. V. (2006). Indicator-displacement assays.

Coordination chemistry reviews, 250(23-24), 3118-3127.

Ngwabebhoh, F. A., \& Yildiz, U. (2019). Nature-derived fibrous

nanomaterial toward biomedicine and environmental remediation: Today's state and future prospects. Journal of Applied Polymer Science, 47878. Diverse Set of Molecules with Experimentally Determined Exposure in the Mouse Brain: Miniperspective. Journal of medicinal chemistry, 60(14), 59435954.

Ström, G., Öhgren, C., \& Ankerfors, M. (2013). Nanocellulose as an additive for foodstuff. Innventia Report, 403, 1-25.

Troszyńska, A., Narolewska, O., Robredo, S., Estrella, I., Hernández, T., Lamparski, G., \& Amarowicz, R. (2010). The effect of polysaccharides on the astringency induced by phenolic compounds. Food quality and preference, 21(5), 463-469.

Tse, W. C., \& Boger, D. L. (2004). A fluorescent intercalator displacement assay for establishing DNA binding selectivity and affinity. Accounts of chemical research, 37(1), 61-69. 
Turbak A. F., Snyder F. W., \& Sandberg K. R. (1982). Food products containing microfibrillated cellulose. United States Patent No. 4341807A. Turbak, A. F., Snyder, F. W., \& Sandberg K. R (1983a) Microfibrillated cellulose, a new cellulose product: properties, uses, and commercial potential. Journal of Applied Polymer Science: Applied Polymer Symposiom, 37, 815827.

Turbak A. F., Snyder F. W., \& Sandberg K. R. (1983b). Suspensions containing microfibrillated cellulose. United States Patent No. 4378381A. Valko, K., Du, C. M., Bevan, C. D., Reynolds, D. P., \& Abraham, M. H. (2000). Rapid-gradient HPLC method for measuring drug interactions with immobilized artificial membrane: Comparison with other lipophilicity measures. Journal of pharmaceutical sciences, 89(8), 1085-1096. Velásquez-Cock, J., Serpa, A., Vélez, L., Gañán, P., Hoyos, C. G., Castro, C., Duizer, L., Goff, H.D., \& Zuluaga, R. (2019). Influence of cellulose nanofibrils on the structural elements of ice cream. Food Hydrocolloids, 87, 204-213.

Yano H., Abe K., Kase Y., Kikkawa S., \& Onishi Y. (2012). Frozen dessert and frozen dessert material. United States Patent No. 20140342075A1. Yuan, M., Liu, Y., Xiao, A., Leng, J., Liao, L., Ma, L., \& Liu, L. (2019). The interaction of dietary flavonoids with xanthine oxidase in vitro: molecular property-binding affinity relationship aspects. RSC advances, 9(19), 1078110788.

Windholz, M. (ed.) (1983). The Merck index. An encyclopedia of chemicals, drugs, and biologicals. $10^{\text {th }}$ edition. Merck \& CO., INC. Rahway, N.J., U.S.A. 
Wood, P. J. (1980). Specificity in the interaction of direct dyes with 492 polysaccharides. Carbohydrate research, 85(2), 271-287. Zhang, J., Umemoto, S., \& Nakatani, K. (2010). Fluorescent indicator displacement assay for ligand- RNA interactions. Journal of the American Chemical Society, 132(11), 3660-3661.

496 
498 Table 1. Binding constants of taste compounds to NFC as mean values with standard deviations $(\mathrm{n}=3)$. Compounds are 499 grouped based on their taste characteristics. For each compounds, molar mass, solubility to water and logP value is 500 provided. negl. $=$ negligible. ${ }^{1}$ Windholz, $1983 ;{ }^{2}$ Furia; $1980 ;{ }^{3}$ Mazzobre, Roman, Mourelle, \& Corti, $2005 ;{ }^{4}$ Dreisewerd, 501 Merz, \& Schembecker, 2015, ${ }^{5}$ Cargill, Inc, 2010; ${ }^{6}$ Valko, Bevan, Reynolds, \& Abraham, 2000; ${ }^{7}$ Yuan, Liu, Xiao, Leng, 502 Liao, Ma, Liu, 2019); ${ }^{8}$ Rankovic, 2017; ${ }^{9}$ Hansch, Leo, \& Hoekman, 1995;

\begin{tabular}{c|c|c|c|c|c|c} 
Taste & Compound & FI & MW & Solubility to & Log P & $\mathbf{K}_{\text {bind, }} \mathbf{M}^{\mathbf{1}}$ \\
& & & {$[\mathbf{g} / \mathbf{m o l}]$} & water $[\mathbf{m g} / \mathbf{m l}]$ & & \\
\hline Sweet & Sucrose & CFW & 342.30 & $2000^{1}$ & $-3.3^{3}$ & negl. \\
& Stevioside & CFW & 804.88 & $1.25^{1}$ & $1.19^{4}$ & $146 \pm 34$ \\
& Aspartame & CFW & 294.31 & $10.20^{2}$ & $0.07^{5}$ & negl. \\
\hline Bitter & Caffeine & CFW (CR) & 194.19 & $21.74^{1}$ & $-0.07^{6}$ & $70 \pm 25(86)$ \\
& Naringin & CFW & 580.53 & $1.00^{1}$ & $-0.5^{7}$ & $1251 \pm 385$ \\
& Quinine & CR & 324.42 & $0.53^{1}$ & $2.51^{8}$ & $14300 \pm 1500$ \\
\hline Umami & Glutamic acid & CFW & 147.13 & $8640^{1}$ & $-3.69^{9}$ & negl.
\end{tabular}


Figure captions

Figure 1. Chemical structures of Cellulose (a), the FIs Calcofluor white (b) and Congo red (c), and the studied taste compounds: aspartame (d), caffeine (e) glutamic acid (f), naringin (g), quinine (h), sucrose (i) and stevioside (j).

Figure 2. Examples of the saturation curves for titration of the FIs with NFC, i.e. the fluorescence intensity of CFW $(6 \mu \mathrm{M})$ (a) and CR $(2.5 \mu \mathrm{M})$ (b) as a function of NFC concentration. The reciprocal plots (Eq. 1, $\theta=\frac{I_{\text {max }}-I_{\text {free }}}{I_{n}-I_{\text {free }}}$ ) are presented in insets.

Figure 3. Examples of the saturation curves for the binding of each taste compound to NFC i.e. the fraction of bound FI as a function of taste compound concentration with estimated trend-lines to help reading. For caffeine (a), both CFW and CR were used as FI. For stevioside (b) and naringin (c) CFW was used as FI and for quinine (d) CR was chosen as FI. Reciprocal plots (Eq. 2, $\alpha=\frac{I_{0}-I_{\text {free }}}{I_{0}-I_{n}}$ ) are presented as insets.

Figure 4. Enthalpy curve of titration of quinine with NFC 